Service social

\title{
Regard sur... les jeunes en Europe centrale et orientale, Sous la direction de Mircea Vultur, Québec, Les Presses de l'Université Laval et Éditions de l'IQRC, 2004, 144 p.
}

\section{Nicole Boucher}

Volume 52, numéro 1, 2006

URI : https://id.erudit.org/iderudit/015956ar

DOI : https://doi.org/10.7202/015956ar

Aller au sommaire du numéro

\section{Éditeur(s)}

École de service social de l'Université Laval

ISSN

1708-1734 (numérique)

Découvrir la revue

\section{Citer ce compte rendu}

Boucher, N. (2006). Compte rendu de [Regard sur... les jeunes en Europe centrale et orientale, Sous la direction de Mircea Vultur, Québec, Les Presses de

l’Université Laval et Éditions de l'IQRC, 2004, 144 p.] Service social, 52(1), 1-2.

https://doi.org/10.7202/015956ar d'utilisation que vous pouvez consulter en ligne.

https://apropos.erudit.org/fr/usagers/politique-dutilisation/ 


\section{RECENSION}

\section{Regard sur... les jeunes en Europe centrale et orientale}

Sous la direction de Mircea Vultur

Québec, Les Presses de l'Université Laval

et Éditions de l'IQRC, 2004, 144 p.

Nicole BOUCHER

Sociologue, professeure

École de service social

Université Laval, Québec

L'ouvrage publié sous la direction de Mircea Vultur, professeur d'économie et de sociologie au centre de recherche Urbanisation, Culture et Société de l'Institut national de la recherche scientifique et membre de l'Observatoire Jeunes et Société, est un petit bijou offert à tous ceux qui s'intéressent à l'évolution de l'Europe centrale et orientale depuis la chute du mur de Berlin.

Parce qu'il couvre globalement de nombreux pays (Roumanie, Russie, Slovaquie, Pologne, Bulgarie, Estonie), parce qu'il aborde la jeunesse de différents points de vue disciplinaires (sociologie, économie, sciences politiques, éducation) et nationaux (Louvain, Budapest, Bucarest, Plovdiv, Slovaquie, Moscou, Paris), parce que la contribution de ces neuf auteurs témoigne d'une connaissance directe et d'un dialogue fructueux avec la société ainsi qu'entre chercheurs, cet ouvrage nous permet de cerner rapidement les difficultés liées à la participation civique des jeunes, le défi qui consiste à assurer une formation scolaire valable lorsque les ressources manquent, le rapport à la religion après une longue absence, les politiques d'emploi ainsi que les itinéraires socioprofessionnels.

Dans cet ouvrage plus descriptif et analytique que théorique, chacun mesure à sa façon les efforts de rapprochement en cours vers la citoyenneté démocratique et vers un mode de vie «occidental». Mais, comme le souligne d'entrée de jeu Siyka Kovacheva, si le chemin à suivre est connu, il n'est pas pour autant facile, considérant, entre autres, le désintérêt des États pour les problèmes de chômage et d'émigration des jeunes.

En effet, nous saisissons rapidement, à la lecture des neuf textes, que les espoirs de la majorité des jeunes d'aujourd'hui des pays de l'Est de parvenir à améliorer leur sort individuellement et collectivement sont souvent déçus en ces temps d'augmentation fulgurante des inégalités sociales. C'est pourquoi, dans l'ensemble, le bilan est plutôt 
négatif quant aux résultats des «transitions » des années 1990, malgré, pour certains pays, leur intégration récente à la communauté européenne. En dépit des efforts personnels dans le secteur du bénévolat, de la politique, de la formation, de l'emploi, dans l'ensemble les auteurs constatent que la situation des jeunes se dégrade nettement. De telle sorte que Julia Subok conclut carrément son article en déclarant : «Ce groupe est le principal perdant des réformes russes et il s'oppose au pouvoir en place » (p. 93).

Dommage qu'aucun auteur n'ait inclus dans son analyse l'importance de l'influence des jeunes de l'Ouest comme modèle pour ceux de l'Est. La mise en évidence d'un constat semblable me semble possible quant à certaines formes de recul et de dégradation pour l'ensemble de la jeunesse dans un contexte de mondialisation auquel ils s'opposent en bloc.

La postface de Gary Bajoit permet de constater toutefois que partout, à l'Est comme à l'Ouest, les jeunes accordent beaucoup d'importance à la famille, à l'école, au travail et à la religion, alors que la méfiance vis-à-vis de la politique est générale. De plus, comme le monde occidental est en train de vivre une véritable mutation de société (p. 137), les jeunes de l'Est sont confrontés de plus en plus à de « nouvelles valeurs », telles que le droit au libre choix, au plaisir, à l'autoréalisation et à la sécurité. Cependant, les opportunités et les moyens dont disposent les jeunes à l'Est sont nettement insuffisants et les frustrations, nombreuses. Voilà probablement pourquoi chacun insiste à sa façon sur l'incertitude qui règne quant à l'avenir et sur des conditions de vie à risques.

Pour nous, Québécois, ces textes qui décrivent l'avant et l'après « rupture » évoquent la Révolution tranquille et la place importante des jeunes durant les «transitions ». À mon avis, le texte d'Alexandru Gurau sur le « renouveau religieux » est particulièrement intéressant et riche d'enseignements quant à l'effet des ruptures sur les jeunes qui sont contraints de se mouvoir dans un contexte de risque exceptionnel (p. 59).

Donc, les jeunes de là-bas pourraient-ils aider les travailleurs sociaux à comprendre les jeunes d'ici? Pourquoi pas! À vous d'en juger en lisant ce petit livre sans prétention et facilement accessible, dont nous ne pouvons malheureusement rendre compte de toute la richesse des articles. Si vous désirez par la suite rendre la comparaison encore plus explicite, je vous recommande, en complément, le visionnement de deux films : Depuis qu'Otar est parti, de Julie Bertuccelli (2002) et Au revoir Lénine!, de Wolfgang Becker (2003). Car le courage au quotidien est nécessaire partout de nos jours.

Merci aux PUL et aux Éditions de l'IQRC de nous faire bénéficier de ce panorama sur les pays de l'Est et sur leurs jeunes des années 2000. 